\title{
Influence du nombre de vehicules d'un convoi sur le comportement dynamique pont-convoi
}

\author{
Abdelouaheb Rezaiguia, Nouredine OuelaA ${ }^{a}$ et Mohamed Guenfoud \\ Laboratoire de Mécanique et Structures, Université de Guelma, BP 401 Guelma, 24000, Algerie
}

Reçu le 10 janvier 2002, accepté le 23 Octobre 2003

\begin{abstract}
Résumé - Nous présentons dans cet article la suite des résultats déjà publiés dans la référence [1]. Cet article traite de la modélisation de pont ferroviaire soumis à des chargements roulants de type convoi. Une modélisation à l'aide de la méthode de superposition modale et du Lagrangien du système est présentée prenant en compte le poids du convoi, les amortisseurs viscoélastiques des roues ainsi qu'un nombre de travées indéterminé. Les équations couplées du mouvement pont-convoi sont résolues numériquement par la méthode de Newmark avec un calcul itératif. Un exemple d'application est traité à la fin de cet article pour étudier l'influence du nombre de véhicules du convoi sur le comportement dynamique du système pont-convoi, et comparer le déplacement et la vitesse verticaux du pont et des véhicules avec et sans amortissement.
\end{abstract}

Mots clés : Comportement dynamique pont-convoi / influence nombre de véhicules / poutre continue / force d'interaction pont-convoi / analyse modale

\begin{abstract}
Influence of the number of convoy vehicles on the dynamic behaviour bridgeconvoy. In this paper we present the extensions of the results published in reference [1]. This paper studies railway bridge modelisation under moving convoy loading. A modelisation introducing modal superposition and system Lagrangian is presented taking into acount the convoy weight, the wheels viscoelastic suspensions as well as an indeterminate number of spans. The equations of motion for bridge-convoy interaction are numericaly solved by the Newmark's method by means of an iterative procedure. An application exemple provides at the end of this paper regarding the influence of the vehicles number in a convoy on the dynamic behavior of the bridge-convoy system, and a comparison between the vertical displacement and velocity of the bridge and vehicles with and without damping.
\end{abstract}

Key words: Dynamic behaviour bridge-convoy / influence number of vehicles / multi-span beam / interaction force bridge-convoy / modal analysis

\section{Introduction}

Depuis environ un siècle, plusieurs recherches ont été menées pour étudier la dynamique des ponts et pour modéliser la force d'interaction pont-convoi. Les premières études ont été consacrées au développement de solutions analytiques pour des ponts à une seule travée excités par des cas simples de forces en mouvement [2,3]. Avec le développement de l'outil informatique, les chercheurs ont développé des solutions numériques pour résoudre les problèmes de ponts excités par des forces [4-7], ou des masses [8-10] mobiles. Dans l'article [11], les auteurs ont étudié le comportement de poutres continues excitées par des forces mobiles. L'étude

\footnotetext{
a Auteur correspondant : n_ouelaa@yahoo.fr
}

est basée sur la méthode de rigidité dynamique avec l'intégration des équations de mouvement dans l'espace des fréquences par la transformation de Fourier. Dans l'article [12], les auteurs utilisent la méthode de condensation dynamique pour développer un élément d'interaction pont-véhicule d'un pont ferroviaire posé sur appuis simples lors du passage d'un convoi. Dans l'article [13], on trouve une étude récente sur l'interaction poutre-véhicule avec la prise en compte de l'effet de séparation et d'impact entre la poutre et le véhicule mobile. Dans cette étude, la détermination des fréquences et des modes propres de vibration du pont est basée sur la méthode de rigidité dynamique. Les résultats sont présentés pour une poutre sur appuis simples excitée par un modèle de véhicule à deux degrés de liberté en mouvement. Ils ont étudié l'influence des défauts de forme de la voie de roulement sur le 


\section{Nomenclature}

\begin{tabular}{|c|c|}
\hline$a$ & accélération de passage du convoi $\left[\mathrm{m} \cdot \mathrm{s}^{-2}\right]$ \\
\hline$c_{v}$ & amortissement de la suspension du véhicule $v\left[\mathrm{Ns} \cdot \mathrm{m}^{-1}\right]$ \\
\hline$E$ & module d'Young $\left[\mathrm{N} \cdot \mathrm{m}^{-2}\right]$ \\
\hline$E_{c}$ & Énergie cinétique de la poutre \\
\hline$E_{d}$ & Énergie de déformation élastique de la poutre \\
\hline$E_{p}$ & Énergie potentielle due aux appuis intermédiaires \\
\hline$F_{v}$ & force d'interaction exercée par le pont sur la roue du véhicule $v[\mathrm{~N}]$ \\
\hline $\mathrm{g}$ & accélération due à la gravité $\left[\mathrm{m} \cdot \mathrm{s}^{-2}\right]$ \\
\hline$I$ & moment d'inertie du pont $\left[\mathrm{m}^{4}\right]$ \\
\hline$k$ & rigidité des appuis intermédiaires $\left[\mathrm{N} \cdot \mathrm{m}^{-1}\right]$ \\
\hline$k_{v}$ & rigidité de la suspension du véhicule $v\left[\mathrm{~N} \cdot \mathrm{m}^{-1}\right]$ \\
\hline$l$ & longueur du pont $[\mathrm{m}]$ \\
\hline$l_{1}$ & longueur de la travée $[\mathrm{m}]$ \\
\hline$m$ & masse par unité de longueur du pont $\left[\mathrm{kg} \cdot \mathrm{m}^{-1}\right]$ \\
\hline$m_{i j}^{*}$ & composantes de la matrice de masse généralisée \\
\hline$c_{i j}^{*}$ & composantes de la matrice d'amortissement généralisée \\
\hline$k_{i j}^{*}$ & composantes de la matrice de rigidité généralisée \\
\hline$M_{v 1}$ & masse équivalente pour la roue du véhicule $v[\mathrm{~kg}]$ \\
\hline$M_{v 2}$ & masse équivalente pour le véhicule $v[\mathrm{~kg}]$ \\
\hline$n$ & nombre de modes \\
\hline$n v$ & nombre de véhicules \\
\hline$N$ & nombre d'appuis intermédiaires \\
\hline$\omega_{p, i}$ & pulsation propre du pont du mode $i\left[\mathrm{rad} \cdot \mathrm{s}^{-1}\right]$ \\
\hline$\omega_{v}$ & pulsation propre du véhicule $v\left[\mathrm{rad} \cdot \mathrm{s}^{-1}\right]$ \\
\hline$\phi_{i}$ & forme propre du pont (mode $i$ ) \\
\hline$P_{i}^{*}$ & force généralisée du mode $i$ \\
\hline$q_{i}$ & déplacement généralisé du pont (mode $i$ ) \\
\hline$\dot{q}_{i}$ & vitesse généralisée du pont (mode $i$ ) \\
\hline$\ddot{q}_{i}$ & accélération généralisée du pont (mode $i$ ) \\
\hline$r$ & fonction du profil de la voie de roulement $[\mathrm{m}]$ \\
\hline$s_{p}$ & distance entre l'origine et l'appui $p[\mathrm{~m}]$ \\
\hline$t$ & temps $[\mathrm{s}]$ \\
\hline$V$ & vitesse de passage du convoi $\left[\mathrm{m} \cdot \mathrm{s}^{-1}\right]$ \\
\hline$w$ & déplacement verticale du pont $[\mathrm{m}]$ \\
\hline$x$ & position spatiale d'un point sur le pont $[\mathrm{m}]$ \\
\hline$x_{v}$ & position du véhicule $v$ sur le pont $[\mathrm{m}]$ \\
\hline$\xi_{v}$ & facteur d'amortissement dans la susponsion du véhicule $v$ \\
\hline$y_{v 1}$ & déplacement vertical de la roue du véhicule $v[\mathrm{~m}]$ \\
\hline$y_{v 2}$ & déplacement vertical du véhicule $v[\mathrm{~m}]$ \\
\hline$\left[M^{*}\right]$ & matrice de masse généralisée \\
\hline$\left[C^{*}\right]$ & matrice d'amortissement généralisée \\
\hline$\left[K^{*}\right]$ & matrice de rigidité généralisée \\
\hline$\{r\}$ & vecteur des défauts de forme de la voie de roulement \\
\hline$\left\{y_{2}\right\}$ & vecteur des déplacements généralisés des véhicules \\
\hline$\left\{\dot{y}_{2}\right\}$ & vecteur des vitesses généralisées des véhicules \\
\hline$\left\{\ddot{y}_{2}\right\}$ & vecteur des accélérations généralisées des véhicules \\
\hline$\left[M_{2}\right]$ & matrice de masse des véhicules \\
\hline$[C]$ & matrice d'amortissement des véhicules \\
\hline$[K]$ & matrice de rigidité des véhicules \\
\hline$\{q\}$ & vecteur des déplacements généralisés du pont \\
\hline$\{\dot{q}\}$ & vecteur des vitesses généralisées du pont \\
\hline$\{\ddot{q}\}$ & vecteur des accélérations généralisées du pont \\
\hline$[\Phi]$ & matrice modale des $n$ vecteurs propres \\
\hline$\left\{P^{*}\right\}$ & vecteur des forces généralisées \\
\hline
\end{tabular}


facteur d'amplification dynamique et l'effet de séparation et d'impact sur la force d'interaction pont-véhicule.

Nous présentons ici un complément des résultats publiés dans la référence [1] concernant la modélisation vibratoire d'un pont ferroviaire à plusieurs travées lors du passage d'un convoi composé de cinq véhicules. La modélisation tient compte de l'interaction pont-véhicules et des imperfections de la voie de roulement. Le pont est modélisé par une poutre continue, les appuis intermédiaires par des ressorts linéaires de grande rigidité et le convoi par une série de modèles de véhicule à deux degrés de liberté en mouvement. À l'aide de la méthode de superposition modale et du lagrangien du système, on détermine deux équations de mouvement couplées pontconvoi. La résolution de ces équations s'effectue par un classique schéma de Newmark avec calcul itératif.

\section{Modélisation}

Le pont est modélisé par une poutre continue de longueur $l$, de masse linéique $m$, de moment d'inertie $I$ et de module d'Young $E$, simplement appuyée aux extrémités avec $N$ appuis intermédiaires simples (Fig. 1). Le convoi est modélisé par $n v$ véhicules roulants à une vitesse $V$. La masse du véhicule $v$ est notée $M_{v 2}$ et la masse de sa roue $M_{v 1}$. Ces deux masses sont liées par un ressort de raideur $k_{v}$ et un amortisseur de constante d'amortissement $c_{v}$. La position du véhicule $v$ est notée $x_{v}(t)$ et le déplacement vertical du pont $w(x, t)$. Les appuis intermédiaires d'abscisses $x=s_{p}(p=1,2, \ldots, N)$ sont modélisés par des ressorts linéaires de grande rigidité et de même raideur $k$. La position spatiale d'un point sur le pont est notée $x(t)$ et les imperfections de la voie de roulement sont représentées par la fonction $r(x)$. Les déplacements verticaux des masses $M_{v 1}$ et $M_{v 2}$ sont respectivement $y_{v 1}(t)$ et $y_{v 2}(t)$.

\section{1 Équations du mouvement pont-convoi projetées dans la base modale}

Les équations du mouvement pont-convoi projetées dans la base modale sont obtenues en utilisant les équations de Lagrange. Ces équations sont regroupées sous la forme matricielle suivante (voir Annexe) :

$$
\begin{aligned}
& {\left[\begin{array}{cc}
{\left[M^{*}\right]} & {[\Phi]\left[M_{2}\right]} \\
{[0]} & {\left[\mathrm{M}_{2}\right]}
\end{array}\right]\left\{\begin{array}{c}
\{\ddot{q}\} \\
\left\{\ddot{y}_{2}\right\}
\end{array}\right\}+\left[\begin{array}{cc}
{\left[C^{*}\right]} & {[0]} \\
-[C][\Phi]^{T} & {[C]}
\end{array}\right]\left\{\begin{array}{l}
\{\dot{q}\} \\
\left\{\dot{y}_{2}\right\}
\end{array}\right\}} \\
& +\left[\begin{array}{cc}
{\left[K^{*}\right]} & {[0]} \\
-[K][\Phi]^{T}-V[C]\left[\Phi^{\prime}\right]^{T} & {[K]}
\end{array}\right]\left\{\begin{array}{c}
\{q\} \\
\left\{y_{2}\right\}
\end{array}\right\} \\
& =\left\{\begin{array}{c}
\left\{P^{*}\right\} \\
{[K]\{r\}+V[C]\left\{r^{\prime}\right\}}
\end{array}\right\}
\end{aligned}
$$

avec les sous-vecteurs et les sous-matrices

$$
\begin{aligned}
{\left[M^{*}\right]=\left[m_{i j}^{*}(t)\right] ; } & {\left[C^{*}\right]=\left[c_{i j}^{*}(t)\right] ; } \\
& {\left[K^{*}\right]=\left[k_{i j}^{*}(t)\right], i, j=1, \ldots, n }
\end{aligned}
$$

$$
\begin{gathered}
{\left[M_{2}\right]=\operatorname{diag}\left[M_{v 2}\right] ;[C]=\operatorname{diag}\left[c_{v}\right]} \\
{[K]=\operatorname{diag}\left[k_{v}\right], v=1, \ldots, n v} \\
{[\Phi]=\left[\phi_{i}\left(x_{v}(t)\right)\right], i=1, \ldots, n, v=1, \ldots, n v} \\
\left\{P^{*}\right\}=\left\{P_{i}^{*}(t)\right\} ;\{q\}=\left\{q_{i}(t)\right\}, i=1, \ldots, n \\
\{r\}=\left\{r\left(x_{v}(t)\right)\right\} ;\left\{y_{2}\right\}=\left\{y_{v 2}(t)\right\} ; \\
\left\{\dot{y}_{2}\right\}=\left\{\dot{y}_{v 2}(t)\right\} ;\left\{\ddot{y}_{2}\right\}=\left\{\ddot{y}_{v 2}(t)\right\}, \\
v=1, \ldots, n v
\end{gathered}
$$

où les $q_{i}(t)$ sont les coordonnées généralisées à déterminer, $\phi_{i}(x)$ les formes propres de vibration du pont prises à $x=x_{v}(t), y_{v 2}, \dot{y}_{v 2}$, et $\ddot{y}_{v 2}$ sont respectivement le déplacement, la vitesse et l'accélération verticaux de la masse $M_{v 2}$. Les expressions $m_{i j}^{*}, c_{i j}^{*}, k_{i j}^{*}$ et $P_{i}^{*}$ sont respectivement les masses, les amortissements, les raideurs et les forces généralisées qui sont données par [1] :

$m_{i j}^{*}=m \int_{0}^{l} \phi_{i}(x) \phi_{j}(x) \mathrm{d} x+\sum_{v=1}^{n v} M_{v 1} \phi_{i}\left(x_{v}(t)\right) \phi_{j}\left(x_{v}(t)\right)$

$$
\begin{aligned}
c_{i j}^{*}= & 2 V \sum_{v=1}^{n v} M_{v 1} \phi_{i}\left(x_{v}(t)\right) \phi_{j}^{\prime}\left(x_{v}(t)\right) \\
k_{i j}^{*}= & E I \int_{0}^{l} \phi_{i}^{\prime \prime}(x) \phi_{j}^{\prime \prime}(x) \mathrm{d} x+k \sum_{p=1}^{N} \phi_{i}\left(s_{p}\right) \phi_{j}\left(s_{p}\right) \\
& +\sum_{v=1}^{n v} M_{v 1} \phi_{i}\left(x_{v}(t)\right)\left(V^{2} \phi_{j}^{\prime \prime}\left(x_{v}(t)\right)+a \phi_{j}^{\prime}\left(x_{v}(t)\right)\right)
\end{aligned}
$$

$$
\begin{aligned}
P_{i}^{*}= & -\sum_{v=1}^{n v}\left(\left(M_{v 1}+M_{v 2}\right) g \phi_{i}\left(x_{v}(t)\right)\right. \\
& \left.+M_{v 1} \phi_{i}\left(x_{v}(t)\right)\left(V^{2} r^{\prime \prime}\left(x_{v}(t)\right)+a r^{\prime}\left(x_{v}(t)\right)\right)\right)
\end{aligned}
$$

\subsection{Projection de la force d'interaction pont-véhicule dans la base modale}

L'expression de la force d'interaction du véhicule $v$ projetée dans la base modale et prise à $x=x_{v}(t)$ est [1] :

$$
\begin{gathered}
F_{v}(t)=\left(M_{v 1}+M_{v 2}\right) g+M_{v 2} \ddot{y}_{v 2}(t) \\
+M_{v 1}\left(\sum_{j=1}^{n} \phi_{j}\left(x_{v}(t)\right) \ddot{q}_{j}(t)+2 V \sum_{j=1}^{n} \phi_{j}^{\prime}\left(x_{v}(t)\right) \dot{q}_{j}(t)\right. \\
+\sum_{j=1}^{n}\left(V^{2} \phi_{j}^{\prime \prime}\left(x_{v}(t)\right)+a \phi_{j}^{\prime}\left(x_{v}(t)\right)\right) q_{j}(t) \\
\left.+V^{2} r^{\prime \prime}\left(x_{v}(t)\right)+a r^{\prime}\left(x_{v}(t)\right)\right)
\end{gathered}
$$

D'après (11), la force d'interaction dépend de la vitesse, de l'accélération du véhicule, des imperfections de la voie de roulement et du mouvement vertical du pontconvoi. 


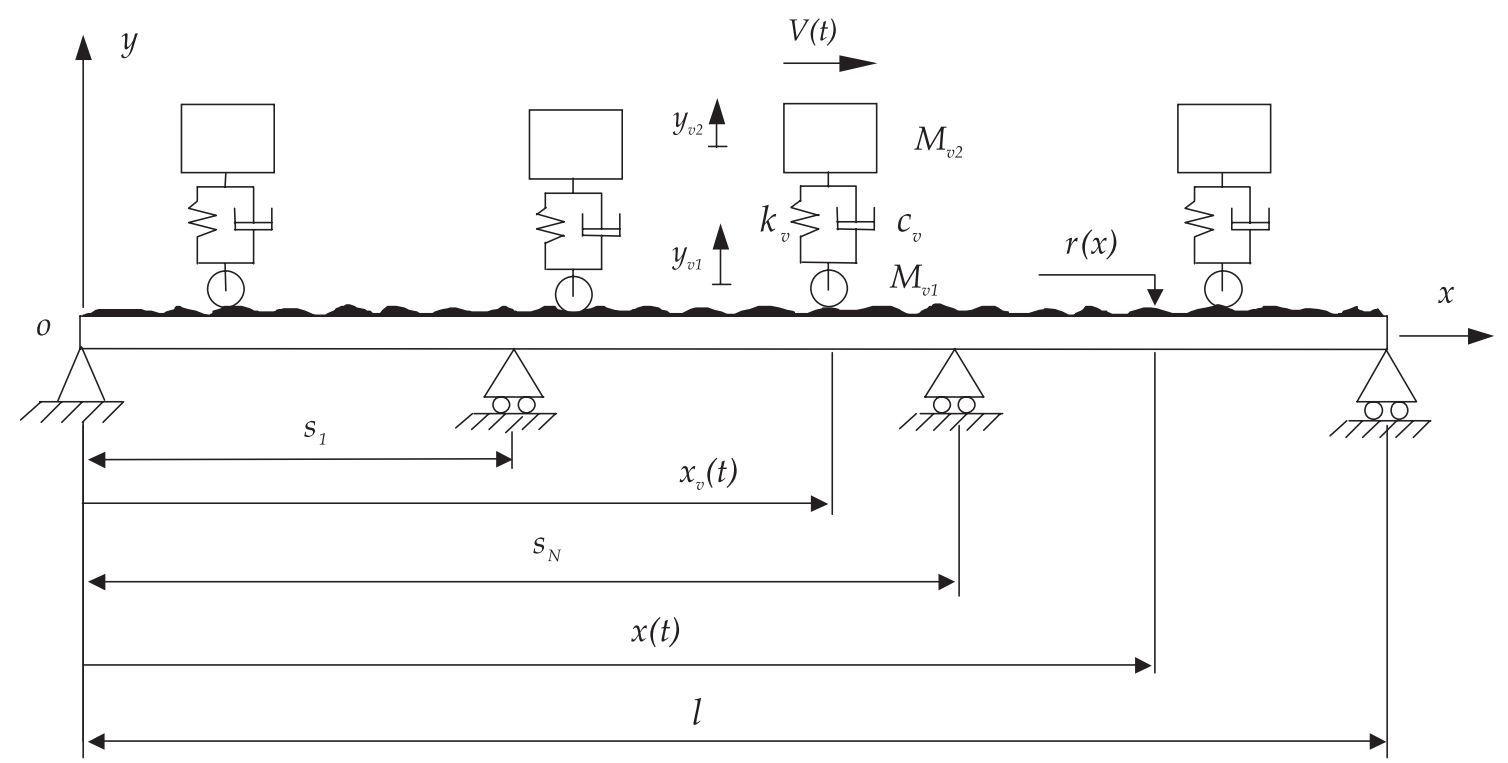

Fig. 1. Modèle mathématique : poutre continue avec $N$ appuis intermédiaires simples, excitée par $n v$ véhicules mobiles.

\section{Résultats et interprétation}

La résolution numérique du système d'équations du mouvement couplé s'effectue en utilisant l'algorithme de l'accélération moyenne de Newmark avec un calcul itératif, sans tenir compte des défauts de forme de la voie de roulement et en conservant la vitesse du roulement du convoi constante. La structure du pont testé est modélisée par une poutre équivalente de longueur $l=60 \mathrm{~m}$, de masse par unité de longueur $m=34088 \mathrm{~kg} \cdot \mathrm{m}^{-1}$, de moment quadratique $I=3,81 \mathrm{~m}^{4}$ et de module d'Young $E=29,43 \times 10^{9} \mathrm{~N} \cdot \mathrm{m}^{-2}$. La modélisation du pont est assez générale pour pouvoir introduire le nombre d'appuis intermédiaires et de véhicules voulus. Les véhicules du convoi étudié sont de masse $M_{v 2}=22000 \mathrm{~kg}$ et $M_{v 1}=$ $220 \mathrm{~kg}$. La raideur et l'amortissement de la suspension viscoélastique sont respectivement, $k_{v}=9 \times 10^{6} \mathrm{~N} \cdot \mathrm{m}^{-1}$ et $c_{v}=1,8 \times 10^{5} \mathrm{Ns} \cdot \mathrm{m}^{-1}$. Les distances entre les véhicules sont supposées uniforme $(12 \mathrm{~m})$. La convergence des résultats numériques dépend du nombre de modes $n$, des conditions aux limites du pont et du nombre d'appuis intermédiaires $N$ et de leurs raideurs $\left(k=10^{9} \mathrm{~N} \cdot \mathrm{m}^{-1}\right)$. Pour un pont simplement appuyé à ses extrémités, la convergence s'effectue pour un nombre de modes $n=2,6$, 8 et 12 pour un nombre d'appuis intermédiaires $N=0,1$, 2 et 3 respectivement. Le nombre d'itérations qui permet la convergence de l'interaction dynamique pont-convoi augmente avec l'augmentation du nombre de véhicules sur le pont et diminue avec l'augmentation de la vitesse de roulement du convoi [14].

Les pulsations propres du pont sont calculées par : $\omega_{p, i}=\gamma_{i}^{2} \sqrt{E I / m l_{1}^{4}}$, avec $\gamma_{i}$ est un paramètre sans dimension qui dépend des conditions aux limites, du nombre de modes et d'appuis intermédiaires [15]. Le tableau 1 montre les six premières pulsations propres du pont en $\mathrm{rad} \cdot \mathrm{s}^{-1}$.
Tableau 1. Pulsations propres du pont.

\begin{tabular}{ccccccc}
\hline & \multicolumn{2}{c}{$N=0$} & \multicolumn{2}{c}{$N=1$} & \multicolumn{2}{c}{$N=2$} \\
\hline Mode & $\gamma_{i}$ & $\omega_{p, i}$ & $\gamma_{i}$ & $\omega_{p, i}$ & $\gamma_{i}$ & $\omega_{p, i}$ \\
\hline 1 & 3,14 & 4,97 & 3,14 & 19,89 & 3,14 & 44,69 \\
2 & 6,28 & 19,88 & 3,92 & 31,07 & 3,55 & 57,27 \\
3 & 9,42 & 44,75 & 6,28 & 79,55 & 4,29 & 83,59 \\
4 & 12,57 & 79,60 & 7,06 & 100,67 & 4,71 & 100,56 \\
5 & 15,71 & 124,33 & 9,42 & 178,97 & 6,70 & 203,65 \\
6 & 18,85 & 179 & 10,21 & 210,07 & 7,43 & 249,92 \\
\hline
\end{tabular}

Les résultats que nous allons présenter montrent l'influence de l'ordre du véhicule dans le convoi et du nombre de véhicules qui compose le convoi sur le comportement dynamique du pont et des autres véhicules (déplacement, vitesse, accélération), ainsi que la force d'interaction pontvéhicules.

\subsection{Influence de l'ordre du véhicule sur le comportement dynamique pont-convoi}

Les figures 2 et 3 montrent l'influence de l'ordre d'un véhicule dans le convoi sur le déplacement et l'accélération verticaux du pont respectivement. On a choisi un convoi de 5 véhicules $(n v=5)$ pour un facteur d'amortissement $\xi_{v}=0$ et une vitesse de roulement $V=30 \mathrm{~m} \cdot \mathrm{s}^{-1}$. Les constatations qu'on peut dégager de ces courbes sont :

1. le déplacement et l'accélération verticaux du pont sont nuls au niveau des appuis intermédiaires correspondant à $s_{1}=l / 3$ et $s_{2}=2 l / 3$, ces appuis sont modélisés par des ressorts linéaires de grande rigidité $k=10^{9} \mathrm{~N} \cdot \mathrm{m}^{-1}$;

2. le déplacement du pont entre les deux appuis intermédiaires sous la roue du véhicule 3 est presque nul, cela s'explique par la symétrie des charges de part et 


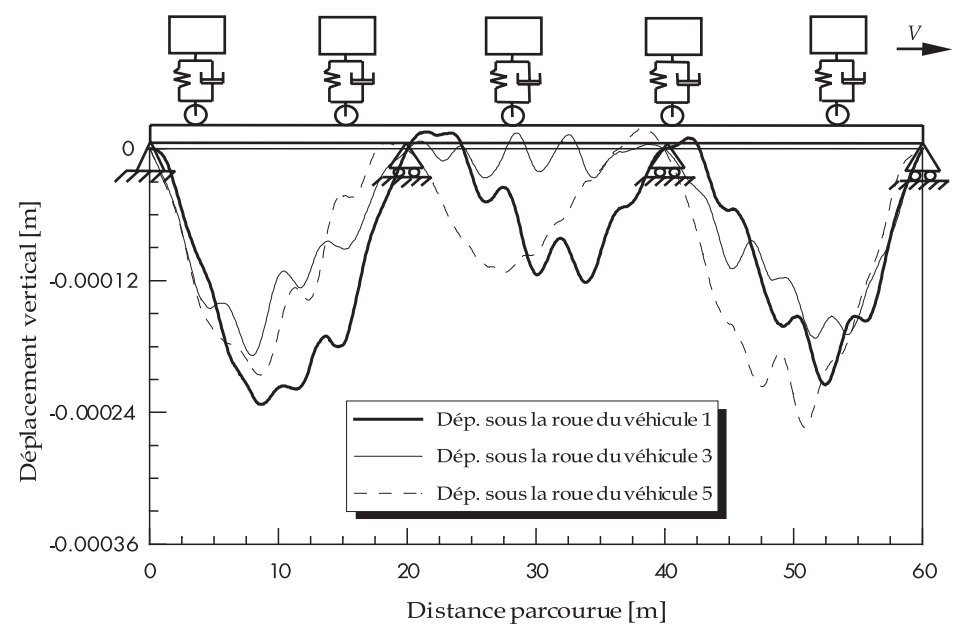

Fig. 2. Déplacement vertical du pont sous chaque roue de véhicules, $\xi_{v}=0, V=30 \mathrm{~m} \cdot \mathrm{s}^{-1}, N=2$.

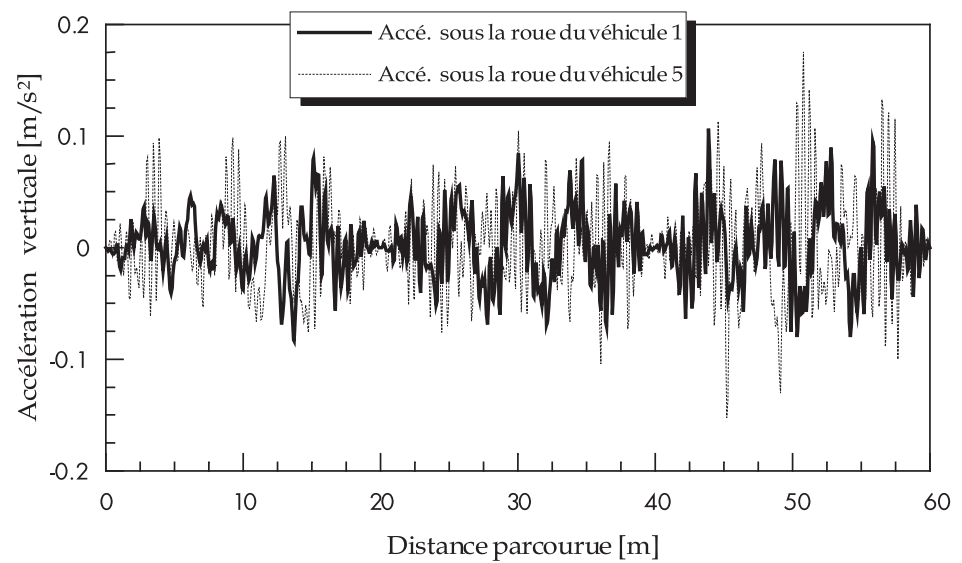

Fig. 3. Accélération verticale du pont sous chaque roue de véhicules, $\xi_{v}=0, V=30 \mathrm{~m} \cdot \mathrm{s}^{-1}, N=2$.

d'autre des appuis intermédiaires correspondant aux véhicules 1,2 et 4,5 ;

3. le déplacement positif situé juste après le premier appui intermédiaire correspond à l'entrée du deuxième véhicule sur le pont, ce phénomène apparaît aussi sur le déplacement du pont sur la roue du cinquième véhicule et cela est dû à la position du quatrième véhicule à droite du deuxième appui intermédiaire.

Pour la figure 3, on constate une faible augmentation des amplitudes des oscillations de l'accélération verticale du pont sous la roue du véhicule 5 , cela est dû à la sortie progressive des véhicules précédents $(1,2,3$ et 4$)$ ce phénomène peut s'expliquer par une excitation réciproque pont-véhicules.

La figure 4 représente les déplacements verticaux des véhicules 1,2 et 5 pour les mêmes conditions que précédemment. On remarque qu'il y a une amplification du déplacement de gauche vers la droite du pont dans le sens de roulement des véhicules.

La figure 5 représente les forces d'interactions en chaque point du contact pont-convoi. Le couplage des équations du pont et du convoi est dû à ces forces d'interactions. On constate que l'amplification des forces d'interactions est plus importante que dans le cas de la figure 4 .

\subsection{Influence du nombre de véhicules sur le comportement dynamique pont-convoi}

Nous présentons respectivement sur les figures 6-8 le déplacement vertical pont-convoi ainsi que la force d'interaction entre eux pour un pont de $25 \mathrm{~m}$ de longueur, sans appuis intermédiaires $(N=0)$ et pour un coefficient d'amortissement $\xi_{v}=0,15$ dans le cas de deux convois l'un composé d'un seul véhicule et l'autre de deux véhicules. On constate sur la figure 6 qu'il y a bifurcation au milieu du pont entre la courbe correspondant au véhicule $1 \mathrm{du}$ convoi composé d'un seul véhicule et la courbe correspondant au véhicule 1 du convoi composé de deux véhicules. Les bifurcations dans le déplacement et la force d'interaction sont dues à l'entrée du deuxième véhicule sur le pont. La troisième courbe représente le déplacement vertical du pont sous la roue du véhicule 2 .

\subsection{Comparaison du comportement dynamique pont-convoi}

Les figures 9 et 10 représentent une comparaison entre le déplacement et la vitesse verticaux pont-convoi pour 


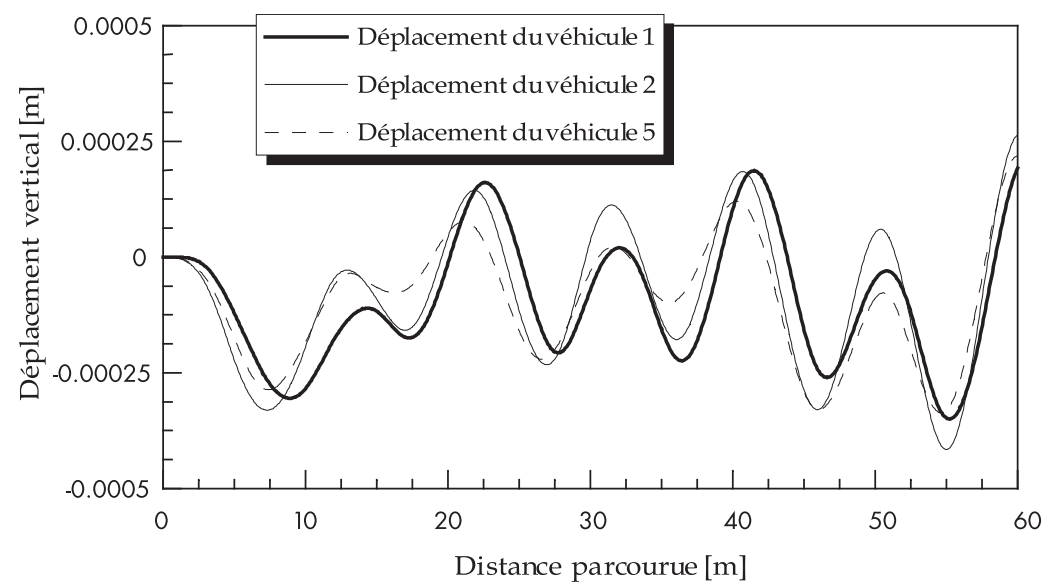

Fig. 4. Déplacement vertical des véhicules, $\xi_{v}=0, V=30 \mathrm{~m} \cdot \mathrm{s}^{-1}, N=2$.

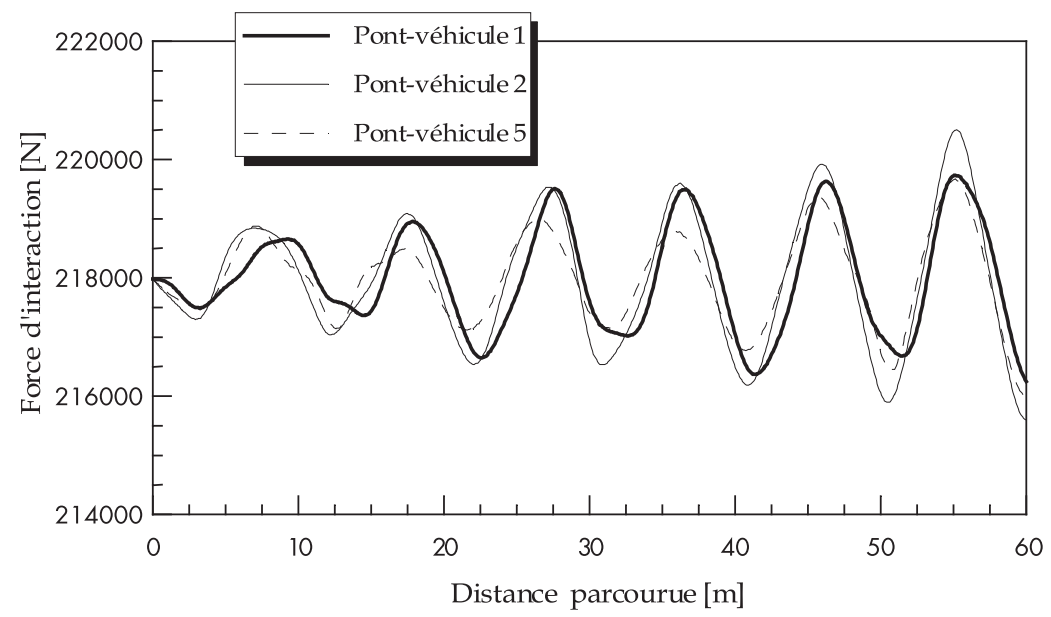

Fig. 5. Force d'interaction en chaque point du contact pont-véhicules, $\xi_{v}=0, V=30 \mathrm{~m} \cdot \mathrm{s}^{-1}, N=2$.

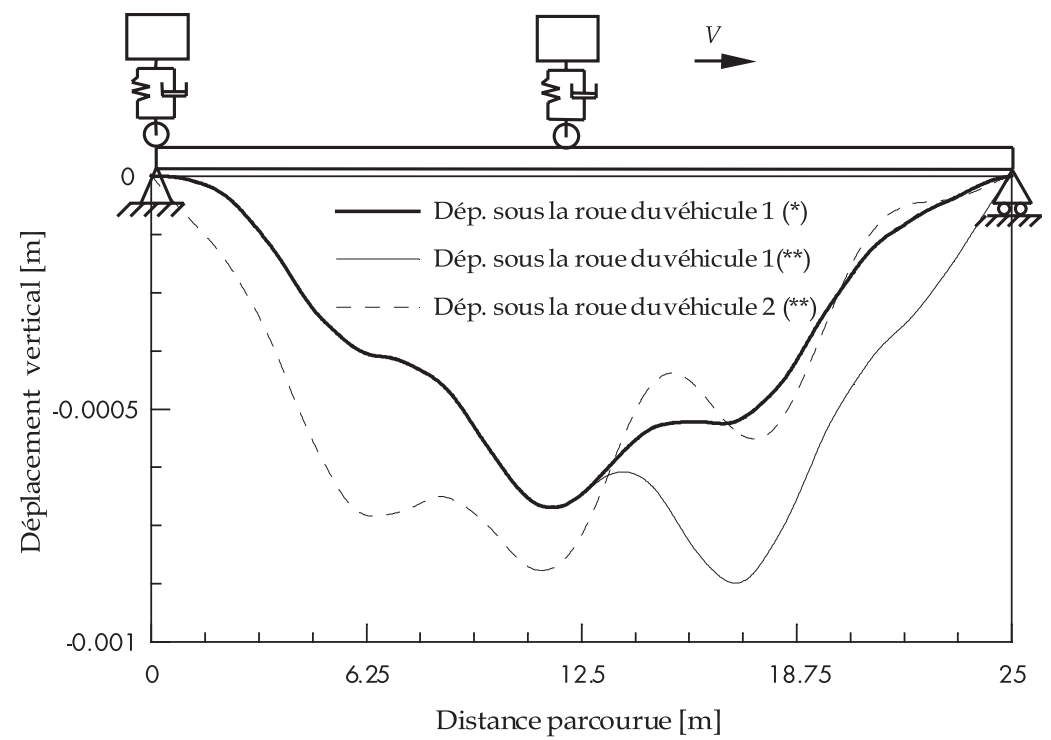

Fig. 6. Influence du nombre de véhicules sur le déplacement vertical du pont sous chaque roue de véhicule, $\xi_{v}=0,15$, $V=30 \mathrm{~m} \cdot \mathrm{s}^{-1}, N=0 .\left(^{*}\right)$ : cas d'un seul véhicule en mouvement, $\left({ }^{*}\right)$ : cas de deux véhicules en mouvement. 


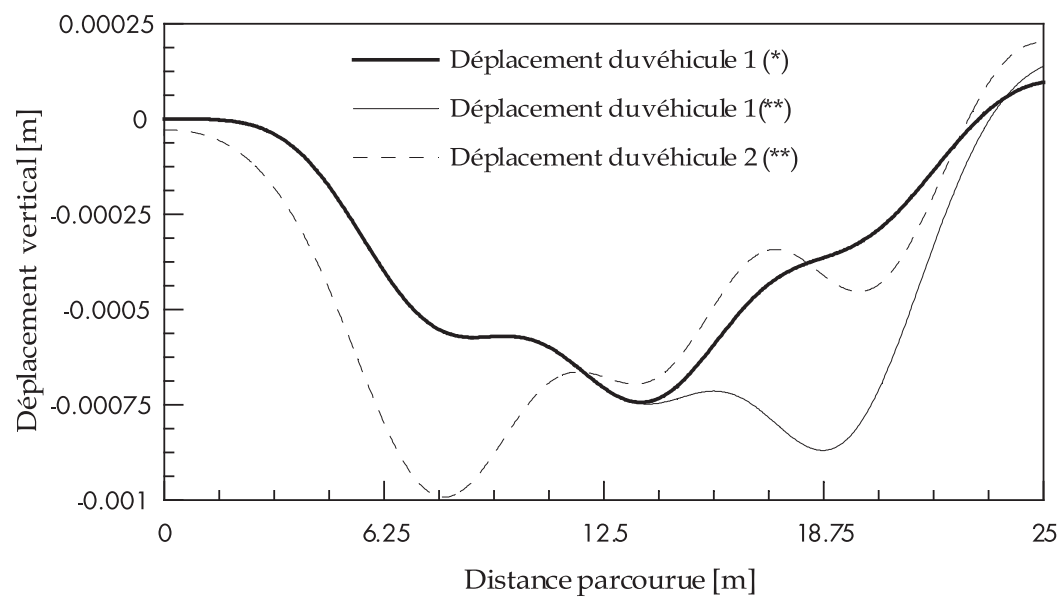

Fig. 7. Influence du nombre de véhicules sur le déplacement vertical des véhicules, $\xi_{v}=0,15, V=30 \mathrm{~m} \cdot \mathrm{s}^{-1}, N=0$ (clé même que Fig. 6).

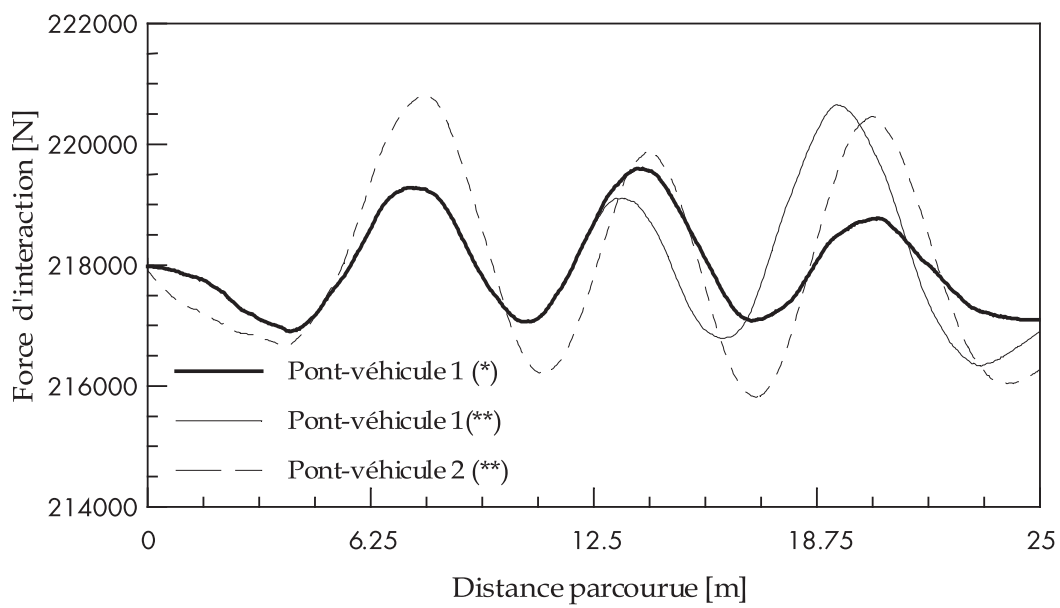

Fig. 8. Influence du nombre de véhicules sur la force d'interaction pont-véhicules, $\xi_{v}=0,15, V=30 \mathrm{~m} \cdot \mathrm{s}^{-1}, N=0$ (clé même que Fig. 6).

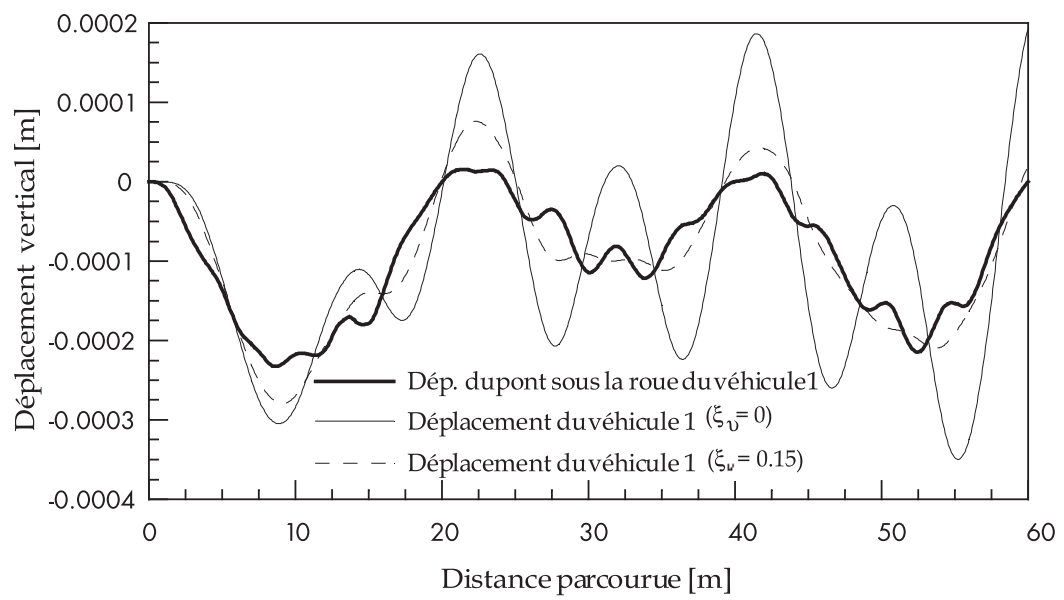

Fig. 9. Comparaison entre les déplacements verticaux du pont sous la roue du véhicule 1 et le déplacement du véhicule 1 , $V=30 \mathrm{~m} \cdot \mathrm{s}^{-1}, N=2$. 


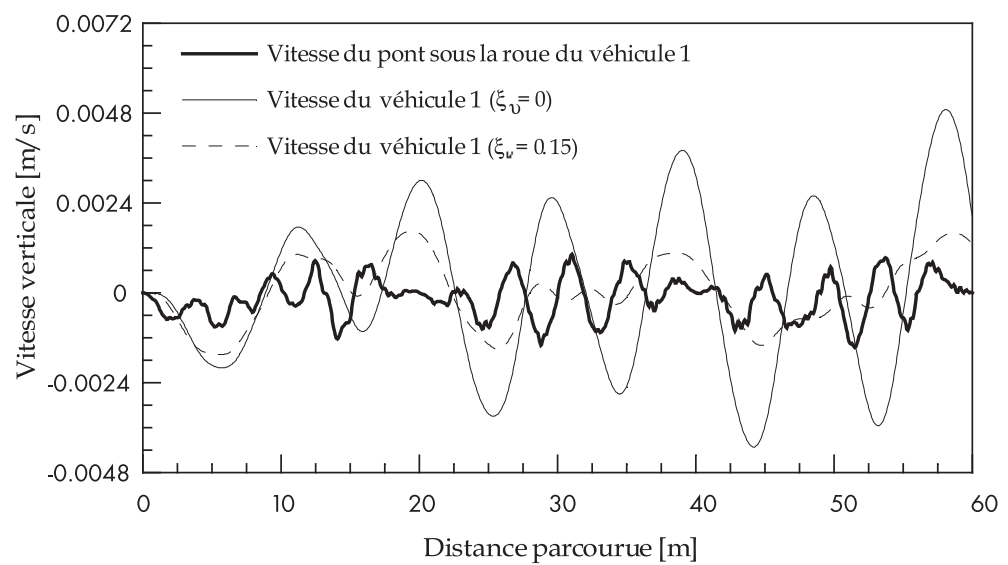

Fig. 10. Comparaison entre les vitesses verticales du pont sous la roue du véhicule 1 et du véhicule $1, V=30 \mathrm{~m} \cdot \mathrm{s}^{-1}, N=2$.

une vitesse de roulement $30 \mathrm{~m} \cdot \mathrm{s}^{-1}$ et pour $\xi_{v}=0$ et $\xi_{v}=0,15$. On constate que le déplacement et la vitesse verticaux du véhicule 1 sont plus importants que ceux du pont et que l'amplitude du déplacement et de la vitesse verticaux du véhicule diminue sensiblement avec l'augmentation de l'amortissement et devient comparable à celle du pont.

\section{Conclusion}

Dans cet article, nous avons présenté les équations essentielles du couplage pont-convoi données explicitement dans $[1,14]$. La résolution numériquement de ces équations est obtenue par la méthode de Newmark avec un calcul itératif. Après l'analyse des résultats obtenus nous avons montré que :

- le nombre de véhicules, qui composent un convoi, influe sur le comportement dynamique des uns sur les autres ainsi que sur le pont;

- la présence d'amortissement visqueux dans la suspension des véhicules diminue sensiblement les amplitudes des déplacements et des vitesses verticaux pontvéhicules.

Les perspectives de cette étude sont d'approfondir l'étude du phénomène d'interaction pont-convoi en tenant compte des défauts de forme du chemin de roulement, modélisation des véhicules par un système à quatre degrés de liberté, détermination de la pression instantanée à partir des accélérations de ce dernier et détermination du facteur d'amplification dynamique.

\section{Annexe : Équations du mouvement pont-convoi}

Pour déterminer les équations du mouvement du pont, nous avons utilisé la méthode modale et les équations de Lagrange. Le déplacement vertical du pont peut être exprimé par :

$$
w(x, t)=\sum_{j=1}^{n} q_{j}(t) \phi_{j}(x)
$$

Le déplacement, la vitesse et l'accélération verticaux de la masse $M_{v 1}$ respectivement sont :

$$
\begin{gathered}
y_{v 1}(t)=w\left(x_{v}(t), t\right)+r\left(x_{v}(t)\right) \\
\dot{y}_{v 1}(t)=\left.\left(\frac{\partial w}{\partial t}+V \frac{\partial w}{\partial x}+V \frac{\mathrm{d} r}{\mathrm{~d} x}\right)\right|_{x=x_{v}(t)}
\end{gathered}
$$

$$
\begin{aligned}
\ddot{y}_{v 1}(t)=\left(\frac{\partial^{2} w}{\partial t^{2}}+\right. & 2 V \frac{\partial^{2} w}{\partial x \partial t}+V^{2} \frac{\partial^{2} w}{\partial x^{2}} \\
& \left.+a \frac{\partial w}{\partial x}+V^{2} \frac{\mathrm{d}^{2} r}{\mathrm{~d} x^{2}}+a \frac{\mathrm{d} r}{\mathrm{~d} x}\right)\left.\right|_{x=x_{v}(t)}
\end{aligned}
$$

L'énergie cinétique et de déformation élastique de la poutre sont données respectivement par :

$$
\begin{aligned}
& E_{c}=\frac{1}{2} \int_{0}^{l} m(x)\left(\frac{\partial w}{\partial t}\right)^{2} \mathrm{~d} x \\
& E_{d}=\frac{1}{2} \int_{0}^{l} E I(x)\left(\frac{\partial^{2} w}{\partial x^{2}}\right)^{2} \mathrm{~d} x
\end{aligned}
$$

Les appuis intermédiaires à $x=s_{p}(p=1,2, \ldots, N)$ sont modélisés par des ressorts de grande rigidité $k$. L'énergie potentielle due aux appuis intermédiaires est donnée par :

$$
E_{p}=\frac{1}{2} k \sum_{p=1}^{N} w^{2}\left(s_{p}, t\right)
$$

En utilisant les équations de Lagrange et compte tenu des expressions (12)-(15), l'équation du mouvement du pont s'écrit :

$$
\begin{aligned}
& \sum_{j=1}^{n} m_{i j}^{*} \ddot{q}_{j}(t)+\sum_{j=1}^{n} c_{i j}^{*} \dot{q}_{j}(t)+\sum_{j=1}^{n} k_{i j}^{*} q_{j}(t) \\
& \quad+\sum_{v=1}^{n v} M_{v 2} \phi_{i}\left(x_{v}(t)\right) \ddot{y}_{v 2}(t)=P_{i}^{*}(t), i=1,2, \ldots, n
\end{aligned}
$$


En appliquant la loi fondamentale de la dynamique, l'équation du mouvement de la masse $M_{v 2}$ s'écrit :

$$
\begin{aligned}
M_{v 2} \ddot{y}_{v 2}(t)+c_{v}\left(\dot{y}_{v 2}(t)\right. & \left.-\dot{y}_{v 1}(t)\right) \\
& +k_{v}\left(y_{v 2}(t)-y_{v 1}(t)\right)=0
\end{aligned}
$$

Compte tenu des expressions (12)-(15), l'équation (20) prend la forme suivante :

$$
\begin{aligned}
M_{v 2} \ddot{y}_{v 2}(t)+c_{v} \dot{y}_{v 2}(t)+k_{v} y_{v 2}(t)-\sum_{j=1}^{n} c_{v} \phi_{j}\left(x_{v}(t)\right) \dot{q}_{j}(t) \\
\quad-\sum_{j=1}^{n}\left(k_{v} \phi_{j}\left(x_{v}(t)\right)+c_{v} V \phi_{j}^{\prime}\left(x_{v}(t)\right)\right) q_{j}(t) \\
=k_{v} r\left(x_{v}(t)\right)+c_{v} V r^{\prime}\left(x_{v}(t)\right), v=1,2, \ldots, n v
\end{aligned}
$$

Regroupons les équations de mouvement (19) et (21) sous la forme matricielle suivante :

$$
\begin{aligned}
& {\left[\begin{array}{cc}
{\left[M^{*}\right]} & {[\Phi]\left[M_{2}\right]} \\
{[0]} & {\left[\mathrm{M}_{2}\right]}
\end{array}\right]\left\{\begin{array}{c}
\{\ddot{q}\} \\
\left\{\ddot{y}_{2}\right\}
\end{array}\right\}+\left[\begin{array}{cc}
{\left[C^{*}\right]} & {[0]} \\
-[C][\Phi]^{T} & {[C]}
\end{array}\right]\left\{\begin{array}{c}
\{\dot{q}\} \\
\left\{\dot{y}_{2}\right\}
\end{array}\right\}} \\
& +\left[\begin{array}{ll}
{\left[K^{*}\right]} & {[0]} \\
-[K][\Phi]^{T}-V[C]\left[\Phi^{\prime}\right]^{T} & {[\mathrm{~K}]}
\end{array}\right]\left\{\begin{array}{c}
\{q\} \\
\left\{y_{2}\right\}
\end{array}\right\}= \\
& \left\{\begin{array}{c}
\left\{P^{*}\right\} \\
{[K]\{r\}+V[C]\left\{r^{\prime}\right\}}
\end{array}\right\}
\end{aligned}
$$

\section{Références}

[1] N. Ouelaa, A. Rezaiguia, M. Guenfoud, Modélisation vibratoire d'un pont à plusieurs travées excité par un convoi en mouvement, Revue Française de Mécanique 1 (2001) 39-47
[2] S.P. Timoshenko, On the forced vibration of bridges, Philosophical Magazine 6 (1922) 1018-1019

[3] L. Fryba, Vibration of solids and structures under moving loads, Groningen, The Netherlands, Noorodhoff International, 1972

[4] A.L. Florence, Traveling force on a Timoshenko beam, ASME, J. Appl. Mech. 32 (1965) 351-358

[5] C.R. Steele, The finite beam with a moving load, ASME, J. Appl. Mech. 34 (1967) 111-118

[6] R. Katz, C.W. Lee, A.G. Ulsoy, R.A. Scott, Dynamic stability and response of a beam subject to a deflection dependent moving load, ASME, Journal of vibration, Acoustics, Stress and Reliability in Design 109 (1987) 361-365

[7] H.P. Lee, Dynamic response of a beam with intermediate point constraints subjected to a moving load, Journal of Sound and Vibration 3 (1994) 361-368

[8] H.D. Nilson, R.A. Conover, Dynamic stability of a beam carrying moving masses, ASME, J. Appl. Mech. 38 (1971) 1003-1006

[9] G.A. Benedetti, Dynamic stability of a beam loaded by a sequence of moving mass particles, ASME, J. Appl. Mech. 41 (1974) 1069-1071

[10] H.P. Lee, Dynamic response of a beam on multiple supports with a moving mass, Structural Engineering and Mechanics 3 (1996) 303-312

[11] K. Henchi, G. Dhatt, M. Talbot, M. Fafard, Dynamic behaviour of multispan beams under moving loads, Journal of Sound and Vibration 1 (1997) 33-45

[12] Y.B. Yang, J.D. Yau, Vehicle-bridge interaction element for dynamic analysis, Journal of Structural Engineering 11 (1997) 1512-1518

[13] Y.S. Cheng, F.T.K. Au, Y.K. Cheung, D.Y. Zheng, On the separation between moving vehicles and bridge, Journal of Sound and Vibration 5 (1999) 781-801

[14] A. Rezaiguia, Comportement dynamique d'un pont de chemin de fer lors du passage d'un convoi. Thèse de Magister, Université de Constantine, Algérie, 2000

[15] Blevins, R.D., Formulas for natural frequency and mode shape, Florida, Krieger Publishing Company, p. 145 